\title{
How far can we expand donor age criteria for pancreas
}

\section{transplantation?}

Jacobus W. Mensink ${ }^{1,2}$, MD, Jacob D. de Boer ${ }^{1}, \mathrm{MD}$, Wouter H. Kopp ${ }^{1}$, MD, Andries E. Braat ${ }^{1}$, MD, PhD

${ }^{1}$ Department of Surgery, Division of Transplantation; Leiden University Medical Center, Leiden

University, Leiden, The Netherlands

${ }^{2}$ Dutch Transplant Foundation, Leiden, The Netherlands

Correspondence to:

Jacobus W. Mensink, MD

Department of Surgery, Division of Transplantation

Leiden University Medical Center

Albinusdreef 2

2333 ZA Leiden

The Netherlands

T. +31715266188

E. j.w.mensink@lumc.nl 


\title{
Conflicts of interest
}

The authors of this manuscript have no conflict of interest. This Letter to the Editor was not prepared or funded in any part by a commercial organization.

\author{
Abbreviations \\ EDC, Extended Donor Criteria \\ EXPAND, Extended Pancreas Donor Program
}

PDRI, Pancreas Donor Risk Index 
With interest, we read the recently published article by Proneth et al on the use of expanded criteria pancreas allografts and we congratulate the authors on the completion of this study. ${ }^{1}$ The study describes 79 pancreas transplantations, with 17 pancreata from donors between 50-60 years old and 1 pancreas from a donor with a Body Mass Index between 30-34 $\mathrm{kg} / \mathrm{m}^{2}$. The authors rightfully conclude that pancreas transplantations with organs from these 'older' donors yield good outcome with similar graft survival.

Due to organ shortage, increasing donor age is observed in all fields of transplantation. For pancreata, professionals are reluctant to accept organs from older donors, mostly due to the increased risk of graft thrombosis and pancreatitis. Despite these risks, the maximum donor age for acceptable pancreata in donation after brain death donors has in some countries already been expanded to 60 years old. ${ }^{2,3}$ Also, increasing numbers of donation after circulatory death pancreas transplantation are reported with excellent outcome. ${ }^{2,4}$ However, as authors state, due to legislation, this is not allowed in Germany.

Key elements incorporated for successful extension of donor limits by this study included local organ procurement to minimize ischemic time and the option to assess organ quality by the local team. ${ }^{1}$ All extended donor criteria (EDC) donors being explanted and transplanted by the 4 participating high-volume centers introduced a substantial bias. As also referred to by the authors, high-volume centers show better graft survival when transplanting pancreata with increased risk factors. ${ }^{5}$ Furthermore, experienced donor surgeons from pancreas transplant centers have a smaller risk of surgical organ injuries during abdominal organ recovery as compared to surgeons from centers not performing pancreas transplantation. ${ }^{6}$ 
The inclusion of only 79 pancreata and 18 EDC pancreata in more than 3 years still suggests a very strict selection, reflected in a minimal extension of donor age with a mean of 51.4 years old in the EDC group. Although being the strongest risk factor, there are more donor factors predicting outcome after transplantation. Axelrod et al weighed and combined these parameters in a continuous scoring tool: the Pancreas Donor Risk Index (PDRI). ${ }^{7}$ If calculated in this study, we hypothesize the PDRI would be about equal for both groups. Again, indicating a very strict donor selection for the EDC group. Therefore, it would have been interesting to also research the number and criteria of pancreata that were not accepted for transplantation within the study period. In general, some regions are more defensive in accepting organs than others. $^{3}$

The Extended Pancreas Donor Program (EXPAND) study shows that pancreata from donors over 50 years old can successfully be used for pancreas transplantation. Again, we congratulate the authors on their efforts to conduct this study and their publication. Hopefully, this initiative will be continued. With a mean donor age of just over 50 years old in the EDC group and the majority of transplantations being performed in low-volume centers, there seem to be options to even further expand the number of pancreas transplantations. 


\section{References}

1. Proneth A, Schnitzbauer AA, Schenker P, et al. Extended Pancreas Donor Program - The EXPAND Study A prospective multicenter trial testing the use of pancreas donors over age 50.

Transplantation. 2018. Transplantation. 2018; 102:1330-1337.

2. Muthusamy ASR, Mumford L, Hudson A, Fuggle SV, Friend PJ. Pancreas Transplantation From Donors After Circulatory Death From the United Kingdom. Am J Transplant. 2013;13(3):824-824.

3. Kopp WH, Vries ED, Boer JD, et al. Donor risk indices in pancreas allocation in the Eurotransplant region. Transpl Internat. 2016;29(8):921-929.

4. Kopp WH, Verhagen MJJ, Blok JJ, et al. Thirty Years of Pancreas Transplantation at Leiden University Medical Center. Transplantation. 2015;99(9).

5. Kopp W, Meel MV, Putter H, et al. Center Volume Is Associated With Outcome After Pancreas Transplantation Within the Eurotransplant Region. Transplantation. 2017;101(6):1247-1253. doi:10.1097

6. Lam HD, Schaapherder AF, Kopp WH, Putter H, Braat AE, Baranski AG. Professionalization of surgical abdominal organ recovery leading to an increase in pancreatic allografts accepted for transplantation in the Netherlands: a serial analysis. Transpl Internat. 2016;30(2):117-123.

7. Axelrod DA, Sung RS, Meyer KH, Wolfe RA, Kaufman DB. Systematic Evaluation of Pancreas Allograft Quality, Outcomes and Geographic Variation in Utilization. Am J Transplant. 2010;10(4):837-845 